\title{
PROCESS AND SYSTEMS The impact of and response to the COVID-19 pandemic on a hospital palliative care team
}

\author{
Authors: Polly M Edmonds, ${ }^{A}$ Katherine E Sleeman, ${ }^{\mathrm{B}}$ Natasha Lovell, ${ }^{\mathrm{C}}$ Rosemary Chester, ${ }^{\mathrm{D}}$ Richard P Towers, ${ }^{\mathrm{E}}$ \\ Stephen A Marshall, ${ }^{\mathrm{F}}$ Irene $]$ Higginson, ${ }^{\mathrm{G}}$ Sabrina Bajwah ${ }^{\mathrm{H}}$ and Wendy Prentice ${ }^{\mathrm{A}}$
}

\begin{abstract}
London was at the forefront of the COVID-19 pandemic in the UK, with an exponential rise in hospital admissions from March 2020. This case study appraises the impact on and response of a hospital palliative care service based in a large inner-city teaching hospital. Referrals increased from a mean of 39 to 75 per week; deaths from 13 to 52 per week. Multiple actions were taken by the team to manage the surge in referrals, which have been categorised based on the $4 \mathrm{~S}$ model: systems, space, stuff and staff. Several lessons are highlighted: need for flexible and responsive staffing over the 7-day week; implementing clear, accessible clinical guidance supported by ward-based teaching; benefits of integrating clinical practice with research; and the importance of maintaining team wellbeing and camaraderie to sustain change. Further evaluation is needed of the differential impact of changes made to inform service planning for future pandemics.
\end{abstract}

KEYWORDS: COVID-19, coronavirus, SARS-CoV-2, palliative care, end-of-life care

DOI: 10.7861/fhj.2020-0131

\section{Introduction}

Since the identification of COVID-19 in Wuhan in December 2019, the pandemic has spread across the world, with over 5 million affected individuals and 351,000 deaths by the end of May 2020.

Authors: ${ }^{\text {A }}$ consultant in palliative medicine, King's College Hospital NHS Foundation Trust, London, UK and Cicely Saunders Institute, London, UK; ${ }^{B}$ clinical scientist, King's College Hospital NHS Foundation Trust, London, UK and Cicely Saunders Institute, London, UK; ' registrar in palliative medicine, King's College Hospital NHS Foundation Trust, London, UK and Cicely Saunders Institute, London,

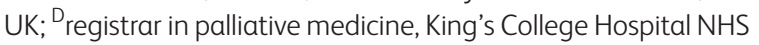

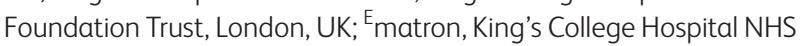
Foundation Trust, London, UK; F social worker, King's College Hospital NHS Foundation Trust, London, UK and Cicely Saunders Institute, London, UK; ' professor of palliative care, King's College Hospital NHS Foundation Trust, London, UK and Cicely Saunders Institute, London, UK; ${ }^{H}$ clinical senior lecturer, King's College Hospital NHS Foundation Trust, London, UK and Cicely Saunders Institute, London, UK
COVID-19 is associated with an increased risk of death in people who are older, male, multimorbid, from Black, Asian and minority ethnic backgrounds and from areas of higher deprivation. ${ }^{2}$ People dying with COVID-19 are affected by symptoms such as breathlessness, agitation and delirium. ${ }^{3}$ Palliative care services provide expertise in symptom relief, clinical decision making and managing uncertainty: all essential components of care in a pandemic. There is emerging data on the palliative care response to the pandemic from the hospice sector. ${ }^{4}$ However, there is little data on the response from hospital palliative care teams. In this case report, we appraise the impact on and response of a hospital palliative care service during the first weeks of the COVID-19 pandemic in the UK.

\section{Impact of the COVID-19 pandemic}

King's College Hospital is part of a large London teaching hospital trust. The site has 1,100 beds and serves an inner-city population of 700,000. The hospital multiprofessional palliative care team is well established, working as an advisory team across the whole hospital. The team provides a visiting service $9 a m-5 p m, 5$ days a week, with $24 / 7$ consultant-led telephone support. There is a limited weekend and public holiday visiting service, provided by one training-grade doctor, working across three acute hospital sites.

The first case of COVID-19 was confirmed in the hospital on 04 March 2020 and the first death was on the 15 March 2020. As of 25 May 2020, there had been 1,585 COVID-19 admissions to the hospital, with 255 deaths and 1,222 discharges.

Between 03 February 2020 and 10 May 2020, 632 patients were referred to the palliative care team. Weekly referrals increased from a mean of 39 in February, to 75 at the peak. Two-hundred and twenty-one patients with confirmed COVID-19 were referred. The number of patients on the palliative care caseload who died increased from a mean of 13 per week in February to 52 per week at the peak (Fig 1).

\section{Service changes in response to the COVID-19} pandemic

Several service changes were rapidly instigated from the week of 16 March 2020 in response to the increasing numbers of patients on the palliative care team caseload, and the needs of patients with severe COVID-19. These have been categorised according to the $4 \mathrm{~S}$ framework of Downar and Seccareccia into systems, staff, stuff and space (supplementary material S1). ${ }^{5}$ 
Fig 1. Palliative care team activity February 2020 - May 2020: referrals and deaths.

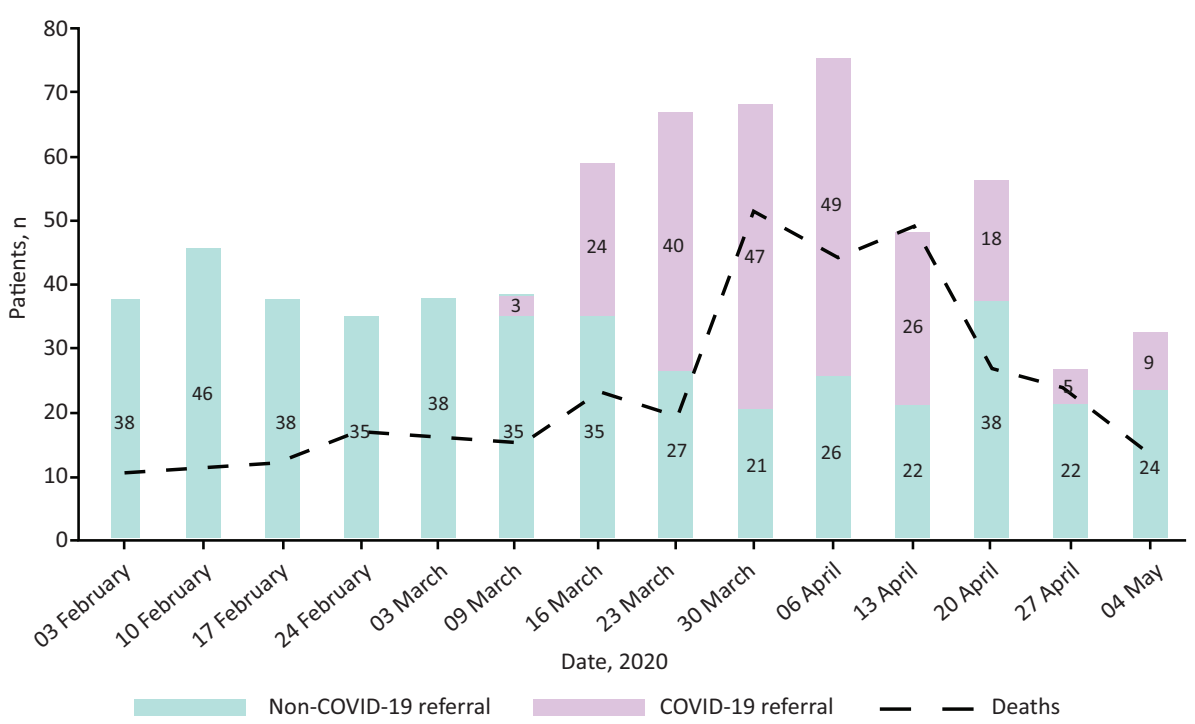

\section{Systems}

System-level changes included integration of the palliative care team into hospital decision-making processes; changes to the palliative care service organisation; rapid development of clinical guidelines and teaching; and strengthened clinical-academic integration. Involvement of the palliative care team in hospital-level decision making facilitated recognition of the essential role of palliative care for people with severe COVID-19. The rapid development of a full 7-day service was essential to balance the flow of work across the whole week; this was a challenge that relied heavily on goodwill, as a relatively small pool of nurse specialists were required to work a minimum of alternate weekends. Development of clinical guidelines for the care of people with COVID-19 was supported by 'bite-sized' teaching. This was delivered to over 500 nurses and junior doctors over a 6 -week period and was particularly valuable for the many staff who had been redeployed at short notice, with little training or experience in care of dying patients. The combination of guidelines and teaching increased staff confidence to start palliative interventions early. Working within an academic department provided flexible clinical cover from clinical academics. It also enabled integration of clinical practice and research (for example, early in the pandemic, clinical team members suggested important research questions relevant to clinical care that the academic team helped answer). ${ }^{3,6,7}$

\section{Space}

Palliative care outpatient clinics were delivered by telephone, facilitated by rapid access to remote IT systems. Video conferencing was used for internal and external meetings. Being co-located in an academic department meant that the clinical team were able to use the spaces made vacant by academic and research teams working from home to ensure social distancing.

\section{Staff}

Facilitating wellbeing and camaraderie among the team was crucial to managing and sustaining the changes required to respond to the pandemic. Twice weekly, socially distanced, exercise sessions; creation of a 'happy board'; and coming together for breaks to relax and debrief were valued and facilitated a 'safe space' in which team members were able to voice their own concerns and anxieties in a rapidly changing situation.

\section{Stuff}

Early in the pandemic, there were isolated instances of lack of syringe pumps and medication. Engagement with medical equipment and pharmacy teams ensured that these issues were not repeated and led to a recognition of the importance of palliative care 'stuff' for optimal patient care.

\section{Areas for improvement}

\section{Systems}

A lack of recognition of palliative care as a frontline service was evident in initial incident planning; this was addressed by proactive representation at divisional meetings, also ensuring rapid communication to and from senior management. Most of the patients referred to palliative care were on medical wards, contrasting with practice prior to the pandemic when a significant number of referrals came from intensive care. This, in part, reflected the nature of intensive care unit deaths from COVID-19, where patients deteriorated rapidly following withdrawal of treatment. The rapid increase in activity for both palliative care and intensive care left limited time for joint planning.

\section{Space and staff}

As a service, our initial response was to meet the needs of patients and families on the wards, with less attention on our own welfare or safety. Implementation of clear social distancing in the office environment and a move to meetings via online platforms were adopted relatively late.

\section{Stuff}

Early in the pandemic, communication around personal protective equipment (PPE) lacked clarity, frequently changed (as national 
guidance evolved) and was focused on distinct clinical areas. This made it difficult to interpret for the team working across the whole site. To mitigate this, we worked with senior management to ensure our own supplies of PPE and scrubs. Although initial planning considered supplies of palliative medication and syringe pumps, early in the pandemic we ran short of both midazolam and syringe pumps. Rapid liaison with pharmacy led to supplies of palliative medicines on COVID-19 wards being prioritised and checked daily and we developed a backup clinical guideline in the event of a critical shortage of midazolam. At one point, a shortage of syringe pumps, prior to the procurement of additional pumps, led to the need for clinical prioritisation of those patients whose symptoms could be managed with regular subcutaneous medication and those that needed an infusion.

\section{Outcomes}

The service changes made during the first weeks of the COVID-19 pandemic enabled the palliative care team to respond to the rapid increase in numbers of patients on the caseload and need for new ways of working. This case study highlights the many levels at which changes occurred, and the interconnectedness between them. Many of the changes implemented are likely to be sustained beyond the acute pandemic, such as the use of virtual technology for meetings; better integration of clinical and academic working; and promotion of mind and body wellbeing within the team. Other important changes (such as the rapid implementation of a full 7-day palliative care service) will not be sustained without additional resource. Involvement of the palliative care team in hospital decision-making processes, as well as focused clinicalacademic outputs, enhanced early recognition of the essential role of palliative care in the pandemic response.,6,7

\section{Conclusion and next steps}

This case report describes how one hospital palliative care service responded to the COVID-19 pandemic. Key findings include:

$>$ the importance of flexible and responsive staffing over the 7-day week to respond to a surge in demand

> implementation of clear, accessible clinical guidance supported by ward-based teaching, and integration of clinical practice with research

$>$ the importance of maintaining team wellbeing and camaraderie to sustain change.

It is essential that there is sufficient capacity within the wider multidisciplinary palliative care team to respond to future surges in demand (for example, from future peaks in the number of people with severe COVID-19). Adequate resourcing of services to provide 7-day care is essential. Future research should identify those changes with maximal impact and sustainability. The importance of palliative care in the pandemic response remains under- recognised. Data on patient outcomes and response to palliative interventions should be gathered to inform the optimal care of people with severe COVID-19.

\section{Supplementary material}

Additional supplementary material may be found in the online version of this article at www.rcpjournals.org/fhj: S1 - Palliative care service response to COVID-19 pandemic.

\section{Funding}

Katherine E Sleeman is funded by a National Institute of Health Research (NIHR) Clinician Scientist Fellowship (CS-2015-15005), Irene J Higginson is an NIHR senior investigator emeritus. Irene ] Higginson is supported by the NIHR Applied Research Collaboration South London (NIHR ARC South London) at King's College Hospital NHS Foundation Trust and by Cicely Saunders International. Irene J Higginson leads the palliative and end-oflife care theme of the NIHR ARC South London, and co-leads the national theme in this.

\section{References}

1 Coronavirus Resource Center. COVID-19 dashboard by the Center for Systems Science and Engineering at Johns Hopkins University. Johns Hopkins University and Medicine. https://coronavirus.jhu. edu/map.html

2 Williamson E, Walker AJ, Bhaskaran K et al. OpenSAFELY: factors associated with COVID-19-related hospital death in the linked electronic health records of 17 million adult NHS patients. medRxiv 2020.05.06.20092999.

3 Lovell N, Maddocks M, Etkind SN et al. Characteristics, symptom management and outcomes of 101 patients with COVID-19 referred for hospital palliative care. J Pain Symptom Manage 2020;60:e77-81.

4 Constantini M, Sleeman KE, Peruselli C, Higginson IJ. Response and role of palliative care during the COVID-19 pandemic: A national telephone survey of hospices in Italy. Pallia Med 2020;34:889-95.

5 Downar J, Seccareccia D. Palliating a pandemic: "all patients must be cared for". J Pain Symptom Manage 2010;39:291-5.

6 Bajwah S, Wilcock A, Towers R et al. Managing the supportive care needs of those affected by COVID-19. Eur Respir ] 2020;55:2000815.

7 Etkind SN, Bone AE, Lovell N et al. The role and response of palliative care and hospice services in epidemics and pandemics: a rapid review to inform practice during the COVID-19 pandemic. J Pain Symptom Manage 2020;60:e31-40.

Address for correspondence: Dr Polly Edmonds, King's College London, Cicely Saunders Institute, Bessemer Road, London SE5 9PJ, UK.

Email:polly.edmonds@nhs.net

Twitter: @CSI_KCL 